\title{
Production, characterization, and cross-reactivity of a polyclonal antibody against Arabidopsis TARGET OF RAPAMYCIN
}

Gyeong-Im Shin ${ }^{1}$, Sun Young Moon ${ }^{2}$, Song Yi Jeong ${ }^{1}$, Myung Geun Ji ${ }^{1}$, Joon-Yung Cha ${ }^{1 *}$ and Woe-Yeon Kim ${ }^{1 *}$ (D)

\begin{abstract}
TARGET OF RAPAMYCIN (TOR), a member of the phosphatidylinositol 3-kinase-related family of protein kinases, is encoded by a single, large gene and is evolutionarily conserved in all eukaryotes. TOR plays a role as a master regulator that integrates nutrient, energy, and stress signaling to orchestrate development. TOR was first identified in yeast mutant screens, as its mutants conferred resistance to rapamycin, an antibiotic with immunosuppressive and anticancer activities. In Arabidopsis thaliana, the loss-of-function tor mutant displays embryo lethality, but the precise mechanisms of TOR function are still unknown. Moreover, a lack of reliable molecular and biochemical assay tools limits our ability to explore TOR functions in plants. Here, we produced a polyclonal a-TOR antibody using two truncated variants of TOR (1-200 and 1113-1304 amino acids) as antigens because recombinant full-length TOR is challenging to express in Escherichia coli. Recombinant His-TOR ${ }^{1-200}$ and His-TOR ${ }^{113-1304}$ proteins were individually expressed in E. coli, and a mixture of proteins (at a 1:1 ratio) was used for immunizing rabbits. Antiserum was purified by an antigen-specific purification method, and the purified polyclonal a-TOR antibody successfully detected endogenous TOR proteins in wild-type Arabidopsis and TOR orthologous in major crop plants, including tomato, maize, and alfalfa. Moreover, our a-TOR antibody is useful for coimmunoprecipitation assays. In summary, we generated a polyclonal a-TOR antibody that detects endogenous TOR in various plant species. Our antibody could be used in future studies to determine the precise molecular mechanisms of TOR, which has largely unknown multifunctional roles in plants.
\end{abstract}

Keywords: Arabidopsis thaliana, Cross-reactivity, Polyclonal antibody, TARGET OF RAPAMYCIN, Truncation

\section{Introduction}

Rapamycin, a natural antibiotic produced by the soil bacteria Streptomyces hyproscopicus, displays antifungal activity through arrest of the cell cycle at the G1 phase and has potent immunosuppressive and cytostatic anticancer activities [1]. It has been reported that rapamycin suppresses the immune system by blocking the cell cycle in T-lymphocytes of mammals [2]. In addition, another natural product, FK506, which is structurally related to rapamycin, has been found as an immunosuppressant,

\footnotetext{
*Correspondence: jycha@gnu.ac.kr; kim1312@gnu.ac.kr ${ }^{1}$ Division of Applied Life Science (BK21 Plus), Plant Molecular Biology and Biotechnology Research Center, Institute of Agricultural and Life Science, Research Institute of Life Science, Gyeongsang National University, Jinju 52828, Republic of Korea

Full list of author information is available at the end of the article
}

and FK506-BINDING PROTEIN 12 (FKBP12) interacts with rapamycin [3].

The Ser/Thr kinase TARGET OF RAPAMYCIN (TOR) was first identified in budding yeast (Saccharomyces cerevisiae) via genetic mutant screening as its mutants confer resistance to rapamycin [4]. TOR, which is encoded by a large gene and is a member of the phosphatidylinositol 3-kinase-related family of protein kinases, is evolutionarily conserved from yeasts to plants and mammals $[5,6]$. TOR is a master regulator that integrates nutrient, energy, and stress signaling to synchronize developmental processes of cells by maintaining a robust rate of ribosome biogenesis and translational initiation [7, 8]. FKBP12 associates with rapamycin, and the complex of FKBP12-rapamycin interacts with the FKBP12-rapamycin-binding (FRB) domain of TOR. 
The rapamycin-FKBP12-TOR ternary complex inhibits the activity of TOR complex 1 (TORC1) in yeast and animals, which is involved in cell growth, development, and proliferation in a temporal manner [9]. In plants, loss of TOR results in embryo lethality; therefore, inducible TOR RNAi lines have been developed to explore the molecular and cellular function of TOR [10, 11]. TOR expresses from early stage to senescence stage, especially in growing tissues [10] and it seems to be involved in stress response as its expression is highly changed upon various stress including drought and UV (http://bar.utoro nto.ca). Moreover, recent studies showed that TOR plays essential roles in plant development, including embryogenesis, seedling growth, root and shoot meristem activation, leaf development, flowering, and senescence [12]. Diverse external and internal upstream signals, such as light, nutrients, starvation, stresses, and phytohormones, mediate TOR signaling by direct translational control of downstream genes [12]. As a kinase, TOR phosphorylates its downstream targets including E2 factors (E2fs; for the cell cycle), S6 kinase (S6K; the cell cycle and mRNA translation), PROTEIN PHOSPHATASE 2A SUBUNIT (TAP46; mRNA translation, $\mathrm{N}$-assimilation, and autophagy), and PYRABACTIN RESISTANCE 1-LIKE (PYL; abscisic acid signaling) [12, 13]. Despite our increased understanding of the roles of TOR in mediating diverse plant developmental processes, molecular and biochemical evidence is still limited due to the lack of reliable assay tools for detecting TOR activity.

Here, we generated two recombinant truncated polypeptides of TOR (1-200 and 1113-1304 amino acids [aa]), which were expressed in Escherichia coli. The purified polypeptides were mixed and injected to rabbits to generated polyclonal TOR antibody, which successfully detected the native full-length Arabidopsis TOR proteins. Notably, our purified polyclonal $\alpha$-TOR antibody has cross-reactivity with its orthologous proteins in crop plants, including tomato (Solanum lycopersicum), maize (Zea mays), and alfalfa (Medicago sativa). Thus, the polyclonal $\alpha$-TOR antibody may enable future studies of the molecular and biochemical functions of TOR.

\section{Materials and methods}

\section{Plant materials and growth conditions}

Arabidopsis thaliana wild-type (Col-0 ecotype) and the estradiol-inducible TOR RNAi line (tor-est) [11] were surface-sterilized, grown in Murashige and Skoog (MS) media, and subsequently transferred to fresh MS media in the absence or presence of estradiol $(10 \mu \mathrm{M})$ for another 5 days. To determine cross-reactivity of the antibody, seeds of Arabidopsis (Col-0), tomato (Solanum lycopersicum), maize (Zea mays), and alfalfa (Medicago sativa) were surface-sterilized, prepared on MS media (for Arabidopsis), B5 agar media (for tomato), or soil (for maize and alfalfa) and grown in a growth chamber for 7 (maize), 8 (Arabidopsis), and 10 days (tomato and alfalfa) under a 16-h-light/8-h-dark photoperiod with a fluorescence rate of $100-120 \mu \mathrm{mol} \mathrm{m}^{-2} \mathrm{~s}^{-1}$ of white light at $23{ }^{\circ} \mathrm{C}$.

\section{Construction and expression of recombinant truncated His-TOR proteins}

Two fragments of TOR, encoding amino acids (aa) 1-200 $\left(\mathrm{TOR}^{1-200}\right)$ and $1113-1304$ TOR $\left.^{1113-1304}\right)$, were cloned into the donor vector $(p C R 8-T O P O)$ and subsequently moved into the $g R S E T$ vector containing a $6 \times \mathrm{His}$ tag (His-) using the recombination-based Gateway cloning system (Invitrogen) according to the manufacturer's instructions. The plasmids were transformed into Escherichia coli BL21 (DE3) for recombinant protein expression, and the transformed cells were grown at $37^{\circ} \mathrm{C}$ until $\mathrm{OD}_{600}=0.6$. After induction by $0.5 \mathrm{mM}$ isoprophyl-1thio- $\beta$-D-galactopyranoside (IPTG), the cells were grown for another $3 \mathrm{~h}$ at $30^{\circ} \mathrm{C}$ and then harvested by centrifugation $(6000 \mathrm{rpm})$ for $10 \mathrm{~min}$. The cells were resuspended in $1 \times \mathrm{PBS}$ and frozen at $-20^{\circ} \mathrm{C}$ until use.

\section{Preparation of antigens and generation of polyclonal antibody in rabbit}

Frozen cells were thawed and incubated with $1 \%(\mathrm{v} / \mathrm{v})$ Triton X-100 for $20 \mathrm{~min}$ before disruption by sonication. Soluble (supernatant [Sup]) and insoluble pellet (precipitate [Ppt]) fractions of His-TOR ${ }^{1-200}$ and His-TOR ${ }^{1113-1304}$ were separated by centrifugation at $12,000 \mathrm{rpm}, 4{ }^{\circ} \mathrm{C}$, for $10 \mathrm{~min}$ and confirmed in $10 \%$ sodium dodecyl sulfate polyacrylamide gel electrophoresis (SDS-PAGE). To obtain sufficient amounts of proteins and produce concentrated antigens, the Ppt fraction was disrupted by several rounds of sonication followed by a treatment with Triton X-100 (1\%). After centrifugation, the Ppt and Sup fractions were redivided. Bands of His-TOR ${ }^{1-200}$ and His-TOR ${ }^{1113-1304}$ were excised from the acrylamide gel, and proteins were eluted from the gel strips using Electro-Eluter (Bio-Rad). Both recombinant proteins of the same concentration were mixed at a ratio of 1:1 (250 $\mu \mathrm{g}$ of His-TOR ${ }^{1-200}: 250 \mu \mathrm{g}$ of His$\mathrm{TOR}^{1113-1304}$ ) and subsequently mixed with Complete Freund's Adjuvant in a 1:1 (v/v) ratio. Prepared antigen was injected into New Zealand White rabbit in the Institutional Animal Care and Use Committee (IACUC)licensed facility at Gyeongsang National University (GNU-171023-B0046). Rabbits were vaccinated with the antigens three times at 15-day intervals. Blood samples were collected from immunized rabbit, and whole serum was collected by centrifugation at $1000 \times g$ for $10 \mathrm{~min}$ at $4{ }^{\circ} \mathrm{C}$. Antiserum was purified using antigen-specific 
affinity purification as described previously [14]. Shortly, insoluble His-TOR ${ }^{1-200}$ and His-TOR ${ }^{1113-1304}$ separated in $10 \%$ SDS-PAGE were transferred onto a polyvinylidene difluoride (PVDF) membrane and the membranes were only excised the portion of His-TOR ${ }^{1-200}$ and HisTOR $^{1113-1304}$ to reduce the non-specific bindings. The blots were blocked with $1 \%(\mathrm{w} / \mathrm{v})$ bovine serum albumin (BSA) in $1 \times$ TBS buffer and subsequently mixed with the antiserum (diluted $1 \mathrm{~mL}$ antiserum in $9 \mathrm{~mL} 1 \%$ BSA) overnight at $4{ }^{\circ} \mathrm{C}$. Blot strips were washed three times with $1 \times$ TBS, sliced into $1 \mathrm{~mm}$ width (for helping antibody elution from blot strips), and bound polyclonal $\alpha$-TOR antibody was eluted with $900 \mu \mathrm{L} 0.1 \mathrm{M}$ glycine (pH 2.5) and immediately neutralized with $100 \mu \mathrm{L} 2 \mathrm{M}$ Tris- $\mathrm{HCl}(\mathrm{pH}$ 8.0). Concentration of the purified antibody is $0.139 \mu \mathrm{g} \mu \mathrm{L}^{-1}$, and stored at $-20^{\circ} \mathrm{C}$ until use.

\section{Immunoblot analysis using purified a-TOR antibody}

Plant tissues were extracted in urea/SDS buffer containing $8 \mathrm{M}$ urea, $5 \%(\mathrm{w} / \mathrm{v}) \mathrm{SDS}, 100 \mathrm{mM}$ Tris $-\mathrm{HCl}(\mathrm{pH} 6.8)$, $1 \mathrm{mM}$ EDTA, 2\% (v/v) $\beta$-mercaptoethanol, $0.00125 \%$ $(\mathrm{w} / \mathrm{v})$ bromophenol blue, and protease/phosphatase/ proteasome inhibitors $\left(1 \mathrm{mM}\right.$ PMSF, $5 \mu \mathrm{g} \mathrm{mL} \mathrm{m}^{-1}$ leupeptin, $1 \mu \mathrm{g} \mathrm{mL} L^{-1}$ aprotinin, $1 \mu \mathrm{g} \mathrm{mL}^{-1}$ pepstatin, $5 \mu \mathrm{g} \mathrm{mL}^{-1}$ antipain, $5 \mu \mathrm{g} \mathrm{mL}{ }^{-1}$ chymostatin, $2 \mathrm{mM} \mathrm{Na}_{2} \mathrm{VO}_{3}, 2 \mathrm{mM}$ $\mathrm{NaF}$, and $50 \mu \mathrm{M}$ MG132). Supernatants after centrifugation $\left(12,000 \mathrm{rpm} / 10 \mathrm{~min} / 4{ }^{\circ} \mathrm{C}\right)$ were separated in $6 \%$ SDS-PAGE and transferred onto a PVDF membrane. Immunoblot analysis was performed using our rabbit polyclonal $\alpha$-TOR antibody (1:500 or 1:1000 dilution), and endogenous TOR protein was detected by chemiluminescence using an ECL-detecting reagent (Thermo Scientific). Nuclear fractionation was isolated using CelLyticTM PN Plant Nuclei Isolation Kit (Sigma-Aldrich) according to the manufacturer's instructions. AntiHSP90 antibody and anti-histone H3 (Abcam) antibody were used as cytosolic and nuclear fraction markers, respectively.

\section{Immunoprecipitation and coimmunoprecipitation}

Proteins of Arabidopsis wild-type and tor-est seedlings, prepared as mentioned above, were extracted in extraction buffer containing $100 \mathrm{mM}$ Tris- $\mathrm{HCl}(\mathrm{pH}$ 7.5), $150 \mathrm{mM} \mathrm{NaCl}, 0.5 \%$ (v/v) NP-40, $1 \mathrm{mM}$ EDTA, $3 \mathrm{mM}$ DTT, and protease/phosphatase/proteasome inhibitors, and the supernatants (Input) were obtained by centrifugation $\left(12,000 \mathrm{rpm} / 5 \mathrm{~min} / 4{ }^{\circ} \mathrm{C}\right)$. Rabbit polyclonal $\alpha$-TOR antibody was incubated with Protein-A agarose (Sigma) for $1 \mathrm{~h}$ at $4{ }^{\circ} \mathrm{C}$ and subsequently mixed with the supernatants for another $3 \mathrm{~h}$ at $4{ }^{\circ} \mathrm{C}$. Beads were washed three times with extraction buffer and resuspended in cold $1 \times$ PBS. Input and coimmunoprecipitated proteins were mixed with the loading buffer and separated in $6 \%$
SDS-PAGE. Immunoblot analysis was performed with the rabbit polyclonal $\alpha$-TOR antibody as described above.

\section{Results and discussion}

Expression and purification of truncated recombinant TOR variants

TOR proteins, evolutionarily conserved in all eukaryotes, possess five domains: HEAT (Huntington, Elongation Factor 3 regulatory, subunit A of PP2A, TOR1) repeats, FAT (FRAP-ATM-TTRAP), FRB, kinase, and FATC (C-terminal FAT domain) (Fig. 1a) [12, 13]. Arabidopsis TOR is a large, single gene encoding 2481 amino acids; thus, recombinant full-length AtTOR is difficult to express in E. coli. To generate a TOR-specific polyclonal antibody, we designed two truncated fragments of TOR, TOR ${ }^{1-200}$ and TOR ${ }^{1113-1304}$ (Fig. 1a). Previously, a synthetic peptide consisting of CTLNRVIADLCSRGNPKEGAP $\left(\mathrm{TOR}^{39-59}\right.$ ) in the HEAT domain was used as a polyclonal TOR antibody and successfully detected native TOR proteins in Arabidopsis [11]. We avoided the kinase domain in TOR to reduce cross-reactivity of our antibody with other various kinase proteins.

Two fragments of TOR were obtained by PCR and cloned into $g R S E T$, which is a His-tagged fusion protein expression vector, by the Gateway system. The two truncated recombinant TOR proteins were individually expressed in E. coli in a small-scale culture $(3 \mathrm{~mL})$, and we found that both truncated recombinant proteins (with estimated sizes of $28 \mathrm{kDa}$ for His-TOR ${ }^{1-200}$ and $26 \mathrm{kDa}$ for His-TOR ${ }^{113-1304}$ ) were highly enriched in the insoluble pellet (Ppt) fractions by $0.5 \mathrm{mM}$ IPTG induction, whereas they were not found in the soluble (Sup) fractions (Fig. 1b, c).

To produce more recombinant protein, the cells expressing each truncated recombinant His-TOR protein were grown in $50 \mathrm{~mL}$ culture, but the concentrations in the Ppt fractions were still low. To concentrate both recombinant proteins, Ppt fractions were disrupted by sonication twice, and the resulting Ppt and Sup fractions were separated by centrifugation (Fig. 2a, b).

To purify His-TOR ${ }^{1-200}$ and His-TOR ${ }^{1113-1304}$, we dissected narrow gel strips corresponding to the portion containing each protein and eluted the proteins from the gel using electro-eluter. Eluted proteins were collected with three fractions from top to bottom, and the estimated concentrations of both His-TOR proteins were approximately $1.5 \mu \mathrm{g} \mu \mathrm{L}^{-1}$ in the top portion, $7.5 \mu \mathrm{g} \mu \mathrm{L}^{-1}$ in the middle, and $15 \mu \mathrm{g} \mu \mathrm{L}^{-1}$ in the bottom, which is normalized by known concentrations of BSA (Fig. 2c). The multisonication step in the middle of antigen preparation increased the concentration of proteins compared to our previous protocols [14]. Thus, we secured 
a

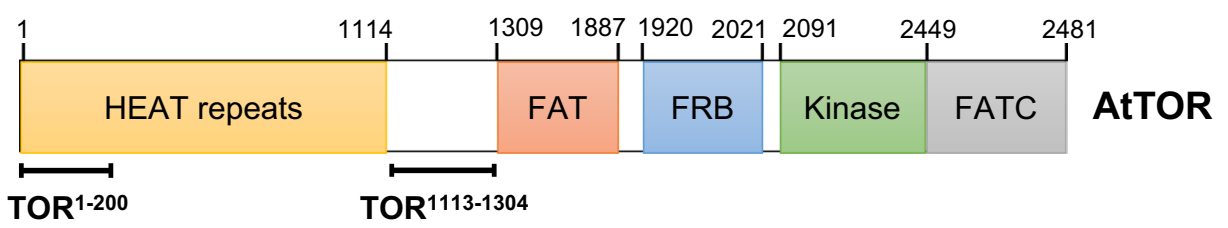

b
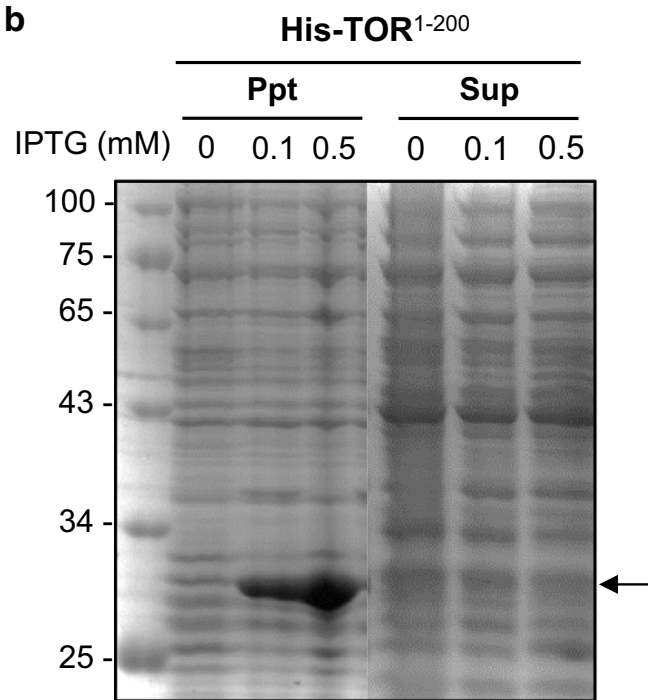

C

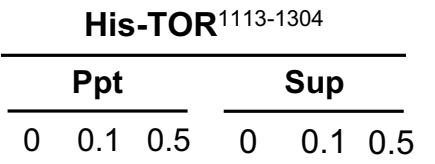

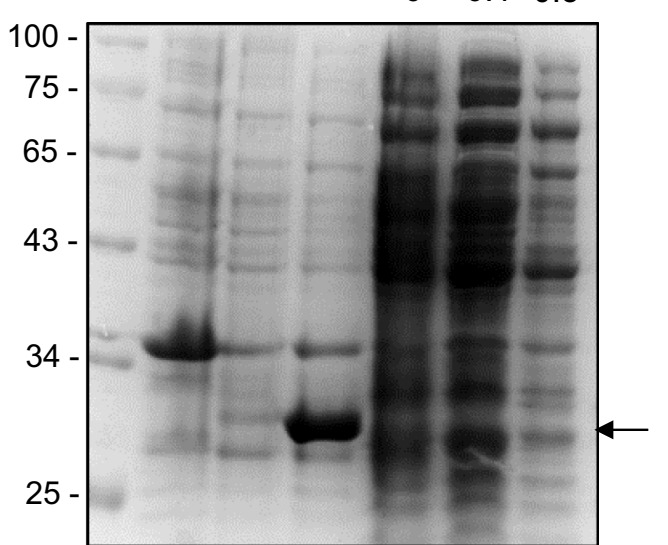

Fig. 1 Design and expression of recombinant truncated Arabidopsis TOR fragments in E. coli. a Schematic representation of Arabidopsis TOR and design of truncated fragments for antigens. Two fragments of TOR, 1-200 aa (TOR ${ }^{1-200}$ ) and $1113-1304$ aa $\left(\right.$ TOR $\left.^{113-1304}\right)$, are shown as bars under the TOR protein. HEAT repeats, Huntingtin, Elongation factor 3, subunit of PP2A, and TOR1; FAT, FRAP-ATM-TRRAP domain; FRB, FKP12-rapamycin binding domain; FATC: C-terminal FAT domain. b, c Expression of recombinant His-TOR ${ }^{1-200}(\mathbf{b})$ and TOR $^{1113-1304}$ (c) proteins in E. coli. Cells harboring gRSET:: TOR ${ }^{1-200}$ and gRSET::TOR ${ }^{1713-1304}$ were individually induced by $0,0.1$, and $0.5 \mathrm{mM} \mathrm{IPTG}$ in a small-scale culture ( $3 \mathrm{~mL}$ LB liquid media). Insoluble pellet (Ppt) and supernatant (Sup) fractions were separated in 10\% SDS-PAGE. Molecular size markers (kDa) are represented on the left. Arrows show the recombinant His-TOR ${ }^{1-200}$ (b) and His-TOR ${ }^{1113-1304}$ (c) proteins

(See figure on next page.)

Fig. 2 Preparation and concentration of recombinant His-TOR ${ }^{1-200}$ and His-TOR ${ }^{1113-1304}$. a Workflow depicting the procedure for producing cell lysates expressing His-TOR ${ }^{1-200}$ and His-TOR ${ }^{1113-1304}$ to concentrate the Ppt fractions. b Preparation of antigens for concentrated Ppt fractions. Ppt fractions containing His-TOR ${ }^{1-200}$ and His-TOR ${ }^{1113-1304}$ isolated from cell lysates were subjected to several rounds of sonication and divided into Ppt and Sup fractions as described in $\mathbf{a}$. The proteins were separated in 10\% SDS-PAGE. c Concentration estimates of His-TOR ${ }^{1-200}$ and His-TOR ${ }^{1113-1304}$. Ppt fractions as prepared in $\mathbf{b}$ were rerun in $10 \%$ SDS-PAGE, and the bands corresponding His-TOR ${ }^{1-200}$ and His-TOR ${ }^{1113-1304}$ were excised from gels. The proteins were eluted from gel strips using the electro-eluter and run in 10\% SDS-PAGE. Eluted proteins were collected by three different fractions (top, middle, and bottom) in a collection tube. BSA was used as a marker protein to estimate the protein concentrations. Molecular size markers $(\mathrm{kDa})$ are represented on the left

sufficient amounts of truncated recombinant TOR proteins for generating polyclonal antibody.

Production and identification of polyclonal a-TOR antibody Previously, we successfully generated polyclonal antibody using a mixture of two truncated fragments of GIGANTEA, a circadian clock controlled flowering time regulator, as an antigen [14]. Based on this, we mixed the two truncated TOR proteins to a final concentration of $500 \mu \mathrm{g}$ using the same concentration of each protein $\left(250 \mu \mathrm{g}\right.$ His-TOR ${ }^{1-200}+250 \mu \mathrm{g}$ His-TOR $\left.{ }^{1113-1304}\right)$ and further mixed with Complete Freund's Adjuvant in a 1:1 (v/v) ratio. Antigen was injected to rabbits three times at 15-day intervals for immunization, and antiserum was collected from blood samples by centrifugation followed by incubation at $4{ }^{\circ} \mathrm{C}$. Membranebased antigen-specific affinity purification was used to purify the polyclonal $\alpha$-TOR antibody from serum 


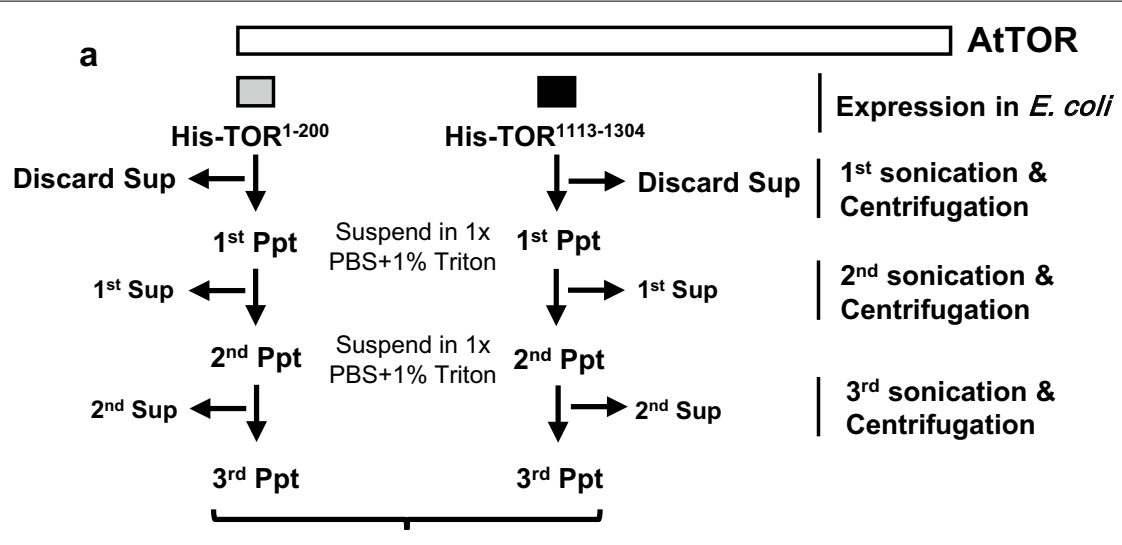

Mix in a 1:1 concentration ratio
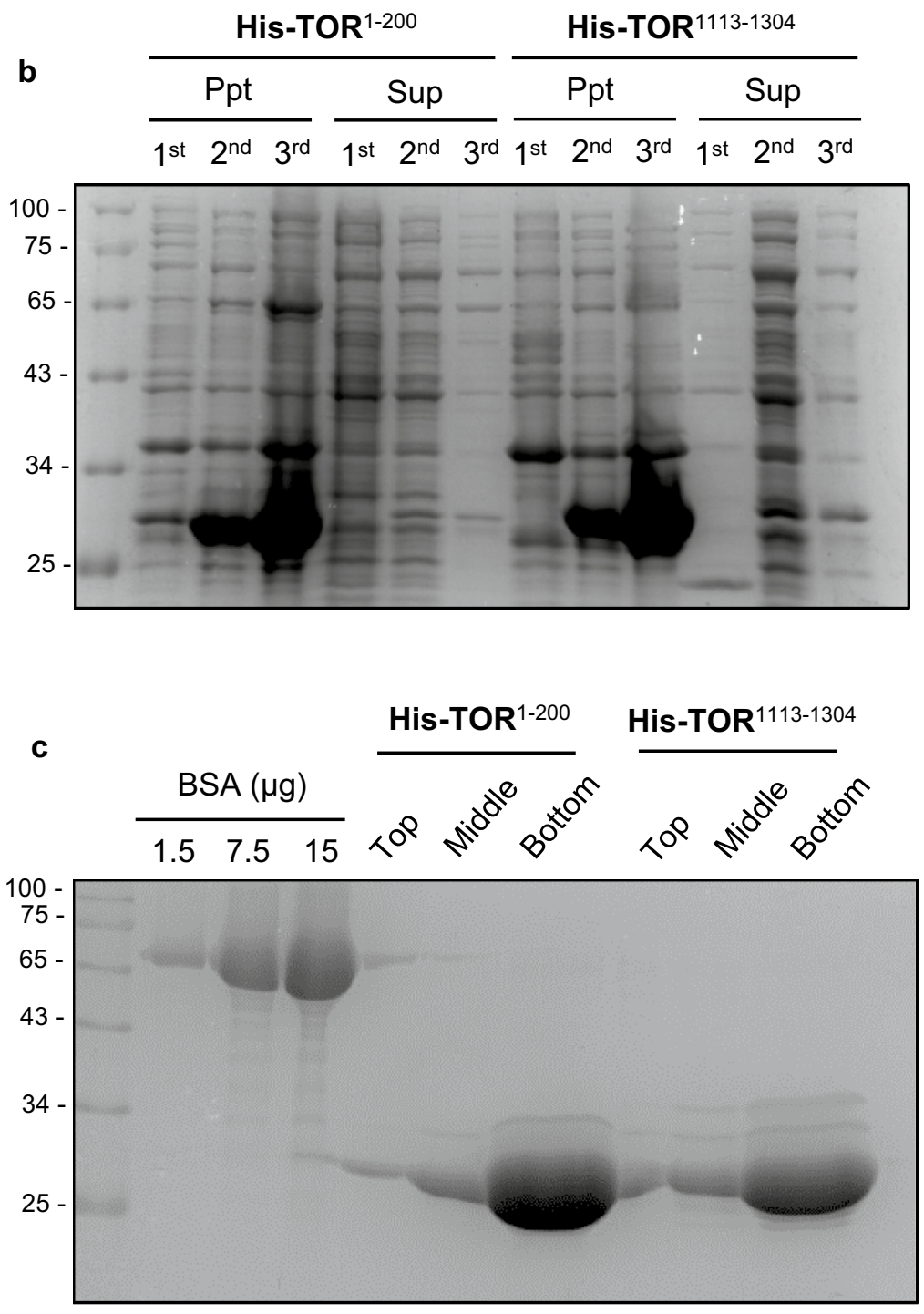
using recombinant His-TOR ${ }^{1-200}$ and His-TOR ${ }^{1113-1304}$ proteins.

Purified polyclonal $\alpha$-TOR antibody was tested using Arabidopsis wild-type (Col-0) plants and an estradiolinducible TOR RNAi (tor-est) line. To induce the silencing of TOR in Arabidopsis, 3-day-old tor-est seedlings grown in MS media were transferred to media with or without estradiol for another 5 days. Based on previous study, tor-est plants in the presence of estradiol displayed dramatic suppression of plant development, as did wildtype plants grown in rapamycin, while in the absence of estradiol or rapamycin, tor-est and wild-type plants grew normally, respectively [11].

Using immunoblot analysis with both whole serum and purified $\alpha$-TOR antibody, we successfully detected endogenous TOR proteins with an approximate size of $280 \mathrm{kDa}$ in wild-type Arabidopsis tissues (Fig. 3a). However, we did not detect TOR proteins in tor-est plants grown in the presence of estradiol, whereas the tor-est plants grown in the absence of estradiol showed similar levels of TOR proteins to that of the wild type (Fig. 3a). Thus, both whole serum and purified antibody could detect native TOR proteins. In addition, we determined the optimum dilution of purified $\alpha$-TOR antibody by testing a dilution of 1:500 and 1:1000 (v/v; antibody:5\% skim milk) and found that TOR proteins were strongly detected in dilutions of 1:500 and also visualized in a dilution of 1:1000 (Fig. 3b). Therefore, we recommend using a working dilution of 1:500 of our purified polyclonal $\alpha$-TOR antibody.

Next, we examined subcellular localization of TOR in Arabidopsis using our polyclonal antibody. Using fractionation protocols, cytoplasmic and nuclear fractions were isolated from wild-type and est-tor plants. We found that TOR is predominantly localized in cytoplasm, but rarely in nucleus (Fig. 3c). Previous study demonstrated that Arabidopsis TOR possesses six putative nuclear localization sequences and localizes in both the cytosol and the nucleus by onion epidermal cell assay [15]. Thus, nuclear-localization of TOR in Arabidopsis needs to be elusive using another detection protocols.

\section{Cross-reactivity of polyclonal a-TOR antibody}

Next, we tried to determine whether our $\alpha$-TOR antibody has cross-reactivity with TOR orthologous in other plants. Due to the physiological importance of TOR in plants, nucleotide and protein sequences of 32 orthologous have been identified in 32 different plant species [16]. Arabidopsis TOR shares 81.2, 80.1, and 72.4\% amino acid sequence identity with that in tomato, alfalfa, and maize, respectively (Additional file 1: Figs. S1, S2). A previous study demonstrated that TOR specifically transmits glucose energy signals in leaves to control the cell

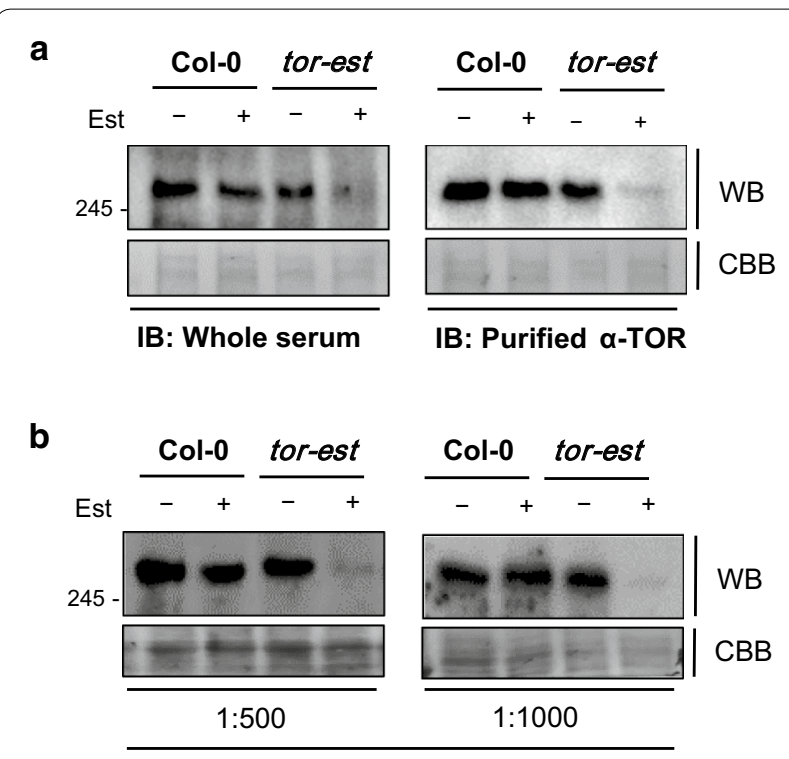

IB: Purified $\alpha-T O R$

C

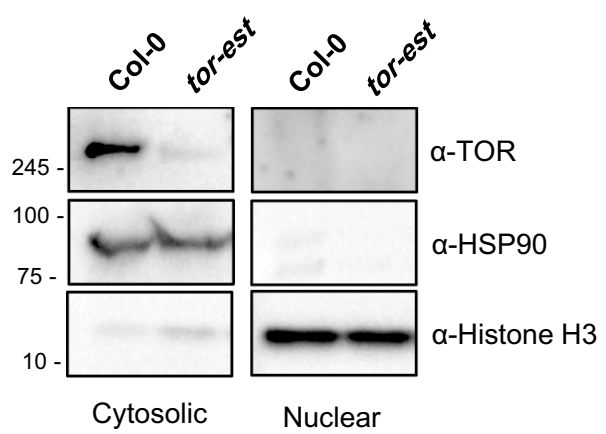

Fig. 3 Confirmation and titration assay using polyclonal a-TOR antibody. a Immunoblot analysis to confirm the specificity of whole serum and purified polyclonal a-TOR antibody. Three-day-old Arabidopsis wild-type and tor-est seedlings were grown in the absence or presence of estradiol for another 5 days. The proteins were separated in 6\% SDS-PAGE and immunoblot analysis was conducted using a 1:500 dilution. b Titration of purified polyclonal a-TOR antibody. The antibody was titrated with 1:500 and 1:1000 dilutions using the same plant extracts shown in $\mathbf{a}$, and immunoblot analysis was conducted. c Subcellular localization of TOR proteins. Cytosolic and nuclear fractions were separated and conducted for immunoblot analysis using a-TOR (1:500), a-HSP90 (1:20,000) as a cytosolic marker, and a-histone H3 (1:3000) as a nuclear marker. Molecular size markers ( $\mathrm{kDa}$ ) are represented on the left. Est, estradiol; $\mathrm{IB}$, immunoblot; CBB, Coomassie brilliant blue-stained blot

cycles in the root meristem [17]. These findings suggest that the TOR kinase plays an essential role in root meristem activity. More recently, it was also reported that TOR mediates the shoot meristem activity by photosynthesisderived light and glucose signals [18]. This suggests that TOR regulates plant growth and development via meristem activity in both roots and shoots. 
Therefore, we tested whether our polyclonal Arabidopsis TOR antibody could not only detect its orthologous proteins in other plants, but also in different tissues such as shoots and roots. First, we tested the cross-reactivity of our polyclonal TOR antibody in Arabidopsis, tomato, maize, and alfalfa. As shown in Fig. 4, our Arabidopsisbased polyclonal TOR antibody successfully detected TOR in young seedlings of Arabidopsis (8 days old) and its orthologous in young seedlings of tomato (10 days old), alfalfa (10 days old), and maize (7 days old), suggesting that our TOR antibody has cross-reactivity with TOR proteins in different plant species. Second, we tested our purified TOR antibody in different tissues and detected TOR in both shoots and roots of Arabidopsis, tomato, alfalfa, and maize (Fig. 4). Arabidopsis TOR is essential for early development; its transcripts are highly expressed in early seedlings displaying strong primary meristem activity [10]. Consistent with this previous report, we detected TOR proteins in all tissues of young seedling plants, regardless of whether they were monocot or dicot plants.

\section{Applications for our polyclonal a-TOR antibody in immunoprecipitation assays}

TOR is identified in all sequenced plants $[19,20]$, and associates with regulatory-associated protein of TOR (RAPTOR) and small lethal with SEC13 protein 8 (LST8) to form TORC1 [21]. TORC1 is regulated by different stimuli such as light, nutrients, phytohormones

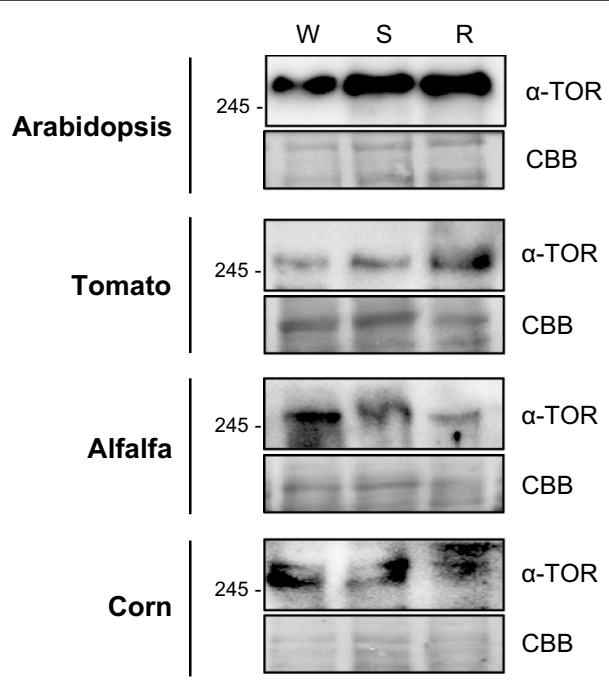

Fig. 4 Cross-reactivity of polyclonal a-TOR antibody in other plant species. The proteins were extracted from whole plants $(W)$, shoots (S), and roots (R) of Arabidopsis (8 days old), tomato (10 days old), alfalfa ( 10 days old), and maize (7 days old) seedlings and separated in 6\% SDS-PAGE. Immunoblot analysis was conducted using purified polyclonal a-TOR antibody (1:500). Molecular size markers (kDa) are represented on the left. CBB, Coomassie brilliant blue-stained blot and stress signal, and phosphorylates and activates downstream signal components [12]. Rho-like small GTPases (GTPase ROPs) in auxin signal and Cauliflower mosaic virus reinitiation factor transactivator-viroplasmin (TAV) increase TOR activity, whereas stress-inducible kinase SnRK1 and SnRK2 repress TOR $[12,13]$. The TOR kinase also regulates multiple downstream proteins involved in the cell cycle, mRNA translation, lipid synthesis, $\mathrm{N}$-assimilation, autophagy, and ABA signaling via phosphorylation $[12,13]$.

To understand the precise molecular mechanisms of TOR in plant development, investigating protein-protein interactions and reciprocal regulation in translational and post-translational levels are essential, and a useful antibody will help to explore unknown substrates. Thus, we tested whether our polyclonal TOR antibody could be used for coimmunoprecipitation assays. Arabidopsis wild-type and tor-est plants were prepared in the absence or presence of estradiol as described in Fig. 3. Extracted proteins were mixed with protein-A agarose beads at $4{ }^{\circ} \mathrm{C}$ for $2 \mathrm{~h}$ following primary binding with our $\alpha$-TOR antibody $\left(4{ }^{\circ} \mathrm{C}\right.$ for $1 \mathrm{~h}$ ). After washing, input and immunoprecipitated proteins probed by $\alpha$-TOR antibody were separated in 6\% SDSPAGE and detected by our $\alpha$-TOR antibody. As shown in Fig. 5, endogenous TOR proteins were immunoprecipitated by our $\alpha$-TOR antibody in wild-type plants (both in the absence and presence of estradiol) and in tor-est plants in the absence of estradiol only, while TOR in tor-est plants grown in the presence of estradiol were not immunoprecipitated because transcripts of TOR are repressed by RNAi in the presence of estradiol, which indicates that endogenous TOR protein is specifically immunoprecipitated by our polyclonal TOR antibody (Fig. 5). Thus, our generated polyclonal TOR antibody could be used for coimmunoprecipitation assays to identify protein-protein interactions.

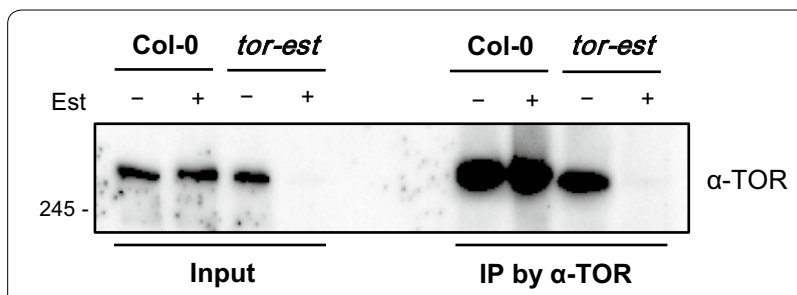

Fig. 5 Immunoprecipitation assay using purified polyclonal a-TOR antibody. Proteins of Arabidopsis wild-type and tor-est seedlings, as prepared in Fig. 3a, were extracted, and the proteins (Input) were mixed with purified polyclonal a-TOR antibody-bound protein A-agarose beads (IP). Input and isolated proteins by IP were separated in 6\% SDS-PAGE, and immunoblot analysis was performed against a-TOR antibody (1:500 dilution). Molecular size markers ( $\mathrm{kDa}$ ) are represented on the left 


\section{Supplementary information}

Supplementary information accompanies this paper at https://doi. org/10.1186/s13765-019-0475-8.

Additional file 1. Additional figures.

\section{Acknowledgements}

Not applicable.

\section{Authors' contributions}

GIS, JYC, and WYK conceived and designed the study. GIS, SYM, SYJ, and MGJ performed the experiments. GIS, JYC, and WYK analyzed the data and wrote the manuscript. All authors critically revised the manuscript. All authors read and approved the final manuscript.

\section{Funding}

This study was supported by grants from the Cooperative Research Program for Agriculture Science \& Technology Development (project no. PJ010953042019), Rural Development Administration, Republic of Korea.

\section{Availability of data and materials}

Not applicable.

\section{Competing interests}

The authors declare that they have no competing interests.

\section{Author details}

${ }^{1}$ Division of Applied Life Science (BK21 Plus), Plant Molecular Biology and Biotechnology Research Center, Institute of Agricultural and Life Science, Research Institute of Life Science, Gyeongsang National University, Jinju 52828, Republic of Korea. ${ }^{2}$ College of Pharmacy and Research Institute of Pharmaceutical Sciences, Gyeongsang National University, Jinju 52828, Republic of Korea.

Received: 17 September 2019 Accepted: 4 November 2019 Published online: 14 November 2019

\section{References}

1. Bierer BE, Mattila PS, Standaert RF, Herzenberg LA, Burakoff SJ, Crabtree G, Schreiber SL (1990) Two distinct signal transmission pathways in T lymphocytes are inhibited by complexes formed between an immunophilin and either FK506 or rapamycin. Proc Natl Acad Sci USA 87:9231-9235

2. Magnuson B, Ekim B, Fingar DC (2012) Regulation and function of ribosomal protein 56 kinase (S6K) within mTOR signaling networks. Biochem J 441(1):1-21

3. Sabatini DM, Erdjument-Bromage H, Lui M, Tempst P, Snyder SH (1994) RAFT1: a mammalian protein that binds to FKBP12 in a rapamycin dependent fashion and is homologous to yeast TORs. Cell 78:35-43

4. Heitman J, Movva NR, Hall MN (1991) Target for cell cycle arrest by the immunosuppressant rapamycin in yeast. Science 253:905-909
5. Horváth BM, Magyar Z, Zhang YX, Hamburger AW, Bakó L, Visser RG, Bachem CW, Bögre L (2006) EBP1 regulates organ size through cell growth and proliferation in plants. EMBO J 25:4909-4920

6. Yuan HX, Xiong Y, Guan KL (2013) Nutrient sensing, metabolism, and cell growth control. Mol Cell 49:379-387

7. Wullschleger S, Loewith R, Hall MN (2006) TOR signaling in growth and metabolism. Cell 124:471-484

8. Dobrenel T, Marchive C, Sormani R, Moreau M, Mozzo M, Montané MH, Menand B, Robaglia C, Meyer C (2011) Regulation of plant growth and metabolism by the TOR kinase. Biochem Soc Trans 39:477-481

9. Yip CK, Murata K, Walz T, Sabatini DM, Kang SA (2010) Structure of the human mTOR complex I and its implications for rapamycin inhibition. Mol Cell 38:768-774

10. Menand B, Desnos T, Nussaume L, Berger F, Bouchez D, Meyer C, Robaglia C (2002) Expression and disruption of the Arabidopsis TOR (target of rapamycin) gene. Proc Natl Acad Sci USA 99:6422-6427

11. Xiang Y, Sheen J (2012) Rapamycin and glucose-target of rapamycin (TOR) protein signaling in plants. J Biol Chem 287:2836-2842

12. Shi L, Wu Y, Sheen J (2018) TOR signaling in plants: conservation and innovation. Development 145:dev160887

13. Schepetilnikov M, Ryabova LA (2018) Recent discoveries on the role of TOR (target of rapamycin) signaling in translation in plants. Plant Physiol 176:1095-1105

14. Khaleda L, Cha J-Y, Kim MG, Kim W-Y (2017) Production and characterization of polyclonal antibody against Arabidopsis GIGANTEA, a circadian clock controlled flowering time regulator. J Plant Biol 60:622-629

15. Ren M, Qiu S, Venglat P, Xiang D, Feng L, Selvaraj G, Datla R (2011) Target of rapamycin regulates development and ribosomal RNA expression through kinase domain in Arabidopsis. Plant Physiol 155:1367-1382

16. Sapre S, Tiwari S, Thakur WV (2018) Phylogenetic analysis of target of rapamycin (TOR) kinase gene of some selected plants species. Biosci Biotech Res Comm 11(3):476-480

17. Xiong Y, McCormack M, Li L, Hall Q, Xiang C, Sheen J (2013) Glucose-TOR signaling reprograms the transcriptome and activates meristems. Nature 496:181-186

18. Pfeiffer A, Janocha D, Dong Y, Medzihradszky A, Schöne S, Daum G, Suzak T, Forner J, Langenecker T, Rempel E, Schmid M, Wirtz M, Hell R, Lohmann $J U$ (2016) Integration of light and metabolic signals for stem cell activation at the shoot apical meristem. eLife 5:17023

19. Yang H, Rudge DG, Koos JD, Vaidialingam B, Yang HJ, Pavletich NP (2013) mTOR kinase structure, mechanism and regulation. Nature 497:217-223

20. Aylett CHS, Sauer E, Imseng S, Boehringer D, Hall MN, Ban N, Maier T (2016) Architecture of human mTOR complex 1. Science 351:48-52

21. Saxton RA, Sabatini DM (2017) mTOR signaling in growth, metabolism, and disease. Cell 168:960-976

\section{Publisher's Note}

Springer Nature remains neutral with regard to jurisdictional claims in published maps and institutional affiliations.

\section{Submit your manuscript to a SpringerOpen ${ }^{\circ}$ journal and benefit from:}

- Convenient online submission

$\checkmark$ Rigorous peer review

- Open access: articles freely available online

- High visibility within the field

- Retaining the copyright to your article

Submit your next manuscript at springeropen.com 